\title{
Diabetes Mellitus Causes Male Reproductive Dysfunction: A Review of the Evidence and Mechanisms
}

\author{
ZUBIN HE ${ }^{1}$, GUOCHAO YIN ${ }^{2}$, QINGDI QUENTIN LI ${ }^{3}$, QIONGFANG ZENG ${ }^{1}$ and JINLIANG DUAN ${ }^{1}$

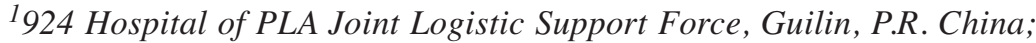 \\ ${ }^{2}$ Guilin Rehabilitation and Recuperation Center, Guilin, P.R. China; \\ ${ }^{3}$ Scientific Review Branch, Division of Extramural Research and Training, \\ National Institute of Environmental Health Sciences, National Institutes of Health, Durham, NC, U.S.A.
}

\begin{abstract}
The metabolic disorders caused by diabetes can lead to various complications, including dysfunction of the male reproductive system. In patients with diabetes, longterm hyperglycemia results in diabetic vascular neuropathy, oxidative stress injury, abnormal zinc metabolism, and insulin resistance syndrome. In addition, insulin deficiency and resistance in diabetes can damage the hypothalamus, pituitary gland, gonads, and perigonads. This can reduce the secretion of sex hormones including gonadotropin-releasing hormone, follicle stimulating hormone, luteinizing hormone, and testosterone, and can lead to testicular atrophy, stromal cell atrophy, seminiferous tubule damage, spermatogenic cell damage, and other structural injuries of the male reproductive organs. These actions can affect male fertility and reproductive health. Herein, we review studies that report a causative role of diabetes in male reproductive function. We also discuss the evidence-based mechanisms involved in the processes of diabetes-related male sexual and reproductive dysfunction as well as the progress in treatment.
\end{abstract}

Diabetes mellitus (DM) is a metabolic disease that seriously endangers human health. The incidence rate of DM is very high, and the age of onset has been decreasing worldwide. The latest data from the International Diabetes Federation (IDF) show the number of adults with diabetes is up to 463

This article is freely accessible online.

Correspondence to: Dr. Jinliang Duan, 924 Hospital of PLA Joint Logistic Support Force, 1 Xinqiaoyuan Road, Xiangshan District, Guilin 541002, P.R. China. Tel: +86 18907738262, e-mail: dj16342@21can.com

Key Words: Diabetes mellitus, male fertility, male sexual dysfunction, male reproductive dysfunction, influencing mechanism, review. million worldwide and is estimated to increase to 578 million by 2030 .

$\mathrm{DM}$ is due to the absolute or relative deficiency of insulin (INS) and insulin resistance (IR), which lead to a decrease in the INS utilization rate and metabolic disorder. In patients with DM, long-term hyperglycemia can cause diabetic vascular neuropathy. Diabetes induced metabolic disorder also leads to oxidative stress, abnormal zinc metabolism, and IR syndrome, all of which affect male fertility and reproductive health. It has been shown that decreased semen quality and impaired reproductive function occur to nearly half of male patients with diabetes. DM incidence is higher in China compared to other countries. The number of male patients with diabetes is gradually increasing, and so are the fertility problems of the male population. Therefore, male reproduction is attracting more and more attention. In this article, we provide an overview and a review of the reproductive pathology of DM males, and evidence on the mechanisms involved in the processes, leading us to conclude that further investigation would benefit our understanding of DM-induced male reproductive dysfunction and would aid in the development of novel ways to improve male reproductive health.

\section{Diabetes Mellitus Affects Male Reproduction}

DM affects male reproduction in four conditions, including erectile dysfunction, ejaculation, structural changes in reproductive organs, and changes in the semen quality.

Studies have found that $59 \%$ of diabetic men have erectile dysfunction (ED). The vast majority of patients with diabetic impotence (DMED) have penile nerve thickening or beaded neuropathy. The decrease in serum testosterone ( $\mathrm{T}$ ) caused by DM also affects vascular endothelial function. Nitric oxide (NO) is an important neurotransmitter in penile erection, and the relaxation effect of $\mathrm{NO}$ on smooth muscle 
cells in corpus cavernosum and microvessels of the penis is at the core of penile erection (1). Hyperglycemia leads to increased levels of reactive oxygen species (ROS), increased advanced glycation end products (AGEs), inhibition of endothelial nitric oxide synthase (eNOS) metabolism, and a decrease in endothelial synthesis and the release of NO, that leads to ED. Neuropathy causes a decrease in NO and an increase in vasoconstrictor endothelin (ET) levels, which are related to erectile function. DM causes metabolic changes in sorbitol metabolism bypass, arteriosclerosis, and disturbance in neurotrophic supply (2), resulting in glycogen deposition, thickening of the basal membrane of somatic nerve sheath cells, and disintegration of axons leading to sensory and motor nerve damage, while the demyelination of pelvic sympathetic nerves leads to the inhibition of sympathetic nerves and eventually DMED (3).

The mechanism of ejaculation is coordinated by pelvic sympathetic nerve fibers. Peripheral neuropathy affects the pelvic sympathetic nerve resulting in the weakening of bladder neck sphincter contraction, while abnormal contraction of the external urethral sphincter leads to the obstruction of semen discharge and return to the bladder, which is called retrograde ejaculation. The appearance of retrograde ejaculation symptoms indicates that diabetic neuropathy is very serious. Unexplained retrograde ejaculation should suggest the possibility of diabetes.

As for the diabetes-induced structural and functional changes in reproductive organs, a decrease in testicular blood flow velocity has been detected in diabetic rats by Doppler (4). It is believed that the decrease in vascular endothelial growth factor (VEGF) expression leads to the impairment of vascular endothelial cell (EC) function, and microcirculation disturbance leads to testicular morphological and structural changes. DM can delay gonadal development in immature rats, decrease sexual behavior and testosterone $(\mathrm{T})$ synthesis, and promote gonadal atrophy. Atrophy of seminiferous tubules (STs), thinning of spermatogenic epithelium, and the rate of empty tubules are higher. In some STs, multinucleated cells with two or three nuclei are found, the vascular degeneration and germ cell apoptosis are increased, and the content of cytoplasmic debris in Sertoli cells near the ST cavity is increased. Ultrastructure has shown that Sertoli cells have irregular basement membranes and the numbers of Sertoli cells is significantly reduced. Also, the oval nuclei in Sertoli cells become irregular and the smooth endoplasmic reticulum in these cells is decreased. Likewise, Leydig cells have irregular nuclei, abundant heterochromatin, and lipid droplets. In addition, the structure of Leydig cell is damaged, its mitochondria are swollen, and endoplasmic reticulum is decreased with dilatation. Liu and colleagues (5) have also found that the amount of sperm in epididymis is decreased significantly, the epididymal epithelium shows vacuole like changes, and a part of the cell nucleus is dissolved and ruptured. Further, mitochondria are found to be transformed into spiral and/or doughnut like organelles, and the number of vesicular organelles is reduced.

The main DM-caused changes in the semen quality are the decrease in sperm density and motility and the increase in sperm DNA fragmentation and apoptosis (6-8). In diabetic rats the mitochondria of spermatogonia show vacuolar changes, the transformation of spermatogonia to primary spermatocytes is decreased, the number of inactive spermatogonia is increased, and the number of epididymal sperm is decreased significantly. DM also affects sperm DNA integrity. Compared with healthy controls, $52 \%$ of men with diabetes have mitochondrial DNA deletions. The increase in sperm DNA fragmentation, advanced glycation end products (AGEs) and receptor for advanced glycation end products (RAGEs), as well as the changes in spermatogenic genes, all lead to the decrease in sperm quality. NO can inactivate superoxide anion, protect sperm membrane from being damaged by lipid peroxide, stabilize cell and lysosomal membranes, increase cGMP content in sperm cells, and facilitate sperm activation and capacitation. NO regulates both sperm motility and sperm lipid peroxidation and affects spermatogenesis. Endothelin (ET) is a vasoconstrictor peptide, which plays an important role in sperm maturation. ET and NO are closely related to male reproductive hormone secretion and spermatogenesis. NO can inhibit the secretion of ET. Deng and coworkers (9) have shown that ET levels in diabetic patients are increased significantly, whereas NO levels are decreased significantly, and ET/NO imbalance leads to endothelial function damage. Due to dyslipidemia, hypertension, and NO synthesis impairment, VEGF expression is decreased, and vascular basement membrane thickening leads to testicular microcirculation disorder, which affects spermatogenesis and capacitation. NOS plays an important role in regulating androgen levels in Leydig cells. INS signaling regulates the number of Sertoli cells as well as testicular size and sperm production. The regulation of the proliferation of immature Sertoli cells by follicle stimulating hormone (FSH) also requires INS signaling pathway. Furthermore, the lack of INSR in Sertoli cells inhibits the activation of the Akt signaling pathway and decreases the expression of the follicle stimulating hormone receptor (FSHR) gene. Some studies have used lentiviral vectors to achieve stable expression of INS in Sertoli cells for the purpose of developing gene therapy (10).

\section{Effect of Oxidative Stress on Male Reproduction in Diabetes Mellitus}

Reactive oxygen species (ROS) is the main cause of cell damage, and one of its main sources is mitochondria. The American Diabetes Association points out that oxidative 
stress (OS) is a common mechanism of diabetic complications, and OS and microcirculation disorders are the main causes of reproductive system damage. OS can cause insulin resistance (IR) by activating protein kinase C (11), $\mathrm{N}$-terminal kinase pathway (12), and transcription factors (13). Long-term blood sugar stimulation leads to upregulation of the number of mitochondria in tissue cells, and lipid peroxidation causes damage to the antioxidant defense system. Long-term hyperglycemia in vivo up-regulates the level of mitochondria in tissue cells. The increase in oxygen radicals and AGEs and their interaction with $\mathrm{NO}$ induces the secretion of peroxynitrite anion (ONOO -) leading to DNA damage. Hyperglycemia then interacts with $\mathrm{NO}$ in vascular endothelial cells to secrete peroxynitrite anion (ONOO -) leading to DNA damage. DNA repair enzymes are then activated in response to DNA damage, and tissue damage is eventually caused by protein kinases and advanced glycation end products (14). Because mitochondrial DNA is almost entirely composed of coding regions, it is easily targeted by ROS because there are no protective mechanisms for mitochondrial DNA. ROS can directly interfere with mitochondrial DNA and RNA replication, resulting in mitochondrial structure damage and dysfunction (15) and decreased glucose oxidation and ATP production, which is a characteristic of IR and hyperglycemia, and mitochondrial damage in turn leads to ROS production. Due to excessive oxidation products, the aldose pathway is activated, and as a result, glucose is converted into sorbitol, nicotinamide adenine dinucleotide phosphate (NADPH) is consumed, and glutathione (GSH) levels are decreased further exacerbating OS. Oxidative damage of islet $\beta$ cells results in their apoptosis and reduction in their number. Testosterone $(\mathrm{T})$ is positively correlated with mitochondrial function and INS sensitivity. In men, the decrease of $\mathrm{T}$ affects mitochondrial function and promotes the occurrence and progress of diabetes (16). $\mathrm{T}$ can regulate the levels of glucose metabolism by protecting mitochondrial function (17), while OS can induce dominant lethal mutations in male gametes and lead to embryo death early in pregnancy.

\section{Effect of Abnormal Zinc Metabolism in Males With Diabetes Mellitus}

Zinc is a powerful antioxidant which can stabilize and maintain the integrity of sperm cell membrane structure by resisting lipid oxidation of sperm membrane. Semen and testis are rich in zinc. Seminal plasma zinc is positively correlated with free $\mathrm{T}$ levels, and zinc regulates $\mathrm{T}$ secretion. The concentration of zinc in islet $\beta$-cells is high, and the highest zinc concentration is within INS containing secretory granules in islet $\beta$-cells. The distribution of zinc in tissues and body fluids of patients with DM is disturbed. Studies have shown that polyuria in type 2 diabetes mellitus (T2DM) may lead to increased excretion of zinc from urine resulting in decrease of plasma zinc concentration. Zinc deficiency may induce SOD mutant conformation and cause endoplasmic reticulum (ER) chronic stress, OS, DNA damage, apoptosis, hypogonadism, spermatogenesis disorder, and sperm morphological defects. Furthermore, the testis and epididymis maintain their function through zinc uptake. Taravati et al. (18) have shown that the concentration of zinc in seminal plasma of asthenospermia is decreased significantly, which suggested the correlation between zinc and sperm motility. During ejaculation, sperm can obtain zinc ions from seminal plasma and improve its motility. Zinc is an important mediator of signal transduction (19) and has been shown to enhance the effect of INS (20). Cellular zinc homeostasis is maintained by a large number of zinc transporters (ZnTs) in cells. ZnTs have been shown to function in reducing cell damage, and zinc homeostasis is precisely regulated by zinc transporters (5). Impaired zinc homeostasis underlies the pathogenesis of many human diseases $(1,2)$. Altered expression of ZnTs not only interferes with zinc homeostasis in organelles, but also leads to organelle dysfunction. In T2DM, the expression of $\mathrm{ZnT}$ genes is significantly reduced. Is there an association between zinc homeostasis and islet $\beta$-cells (21)? All members of the SLC30 family (ZnTs) are expressed in human islet $\beta$-cells, and all ZnTs can be detected in human and mouse $\beta$-cells except ZnT3. ZnT8 is mainly expressed in adult mouse Leydig cells, and the zinc transporters ZnT1$5,7,8$, and 10 have important roles in immune response (5, 22). ZnT8 is also expressed in islet $\beta$-cells. Over-expression of ZnT8 increases the zinc content of $\beta$-cells and promotes glucose-stimulated INS secretion, and ZnT8 also plays a role in $\mathrm{T}$ production through the protein kinase $\mathrm{A}$ (PKA) signaling pathway. ZnT1 is expressed at the plasma membrane of testicular cells, the cytoplasm of spermatogenic cells, and in Leydig cells. In summary, the disturbance of zinc metabolism can diminish male fertility, and the altered $\mathrm{ZnT}$ expression in the gonads of DM patients affects zinc homeostasis.

\section{Role of Hypothalamic Pituitary Gonadal Axis in the Reproduction of Diabetic Men}

Spermatogenesis is regulated by the hypothalamic-pituitarygonadal axis (HPGA), and abnormal HPGA can lead to abnormal spermatogenesis. INS stimulates the expression and secretion of gonadotropin-releasing hormone $(\mathrm{GnRH})$ in the hypothalamus and promotes the function of HPGA (23). In vitro INS also promotes the secretion of gonadotropin (GN) induced by luteinizing hormone-releasing hormone (LHRH). Insufficient secretion of INS in DM patients can damage the HPGA. Glucose metabolism in anterior pituitary cells depends on the secretion of INS. Lack of INS causes 
glucose utilization disorders, which lead to decreased pituitary protein synthesis and decreased GN secretion. Hyperglycemia interferes with the secretion of $\mathrm{GnRH}$, thereby reducing the secretion of GN and prolactin (PRL), which in turn leads to a significant decrease in the secretion of $\mathrm{T}$ from Leydig cells and ultimately to spermatogenesis disorders. Because of the DM-mediated damage of Sertoli cells or Leydig cells, the secretion of $\mathrm{T}$ is decreased resulting in an increase in the levels of FSH and LH through the negative feedback effect of HPGA. INS receptors and INS signaling proteins are widely distributed in the central nervous system. There is an important relationship between brain INS signal transduction and reproduction. INS is a messenger linking metabolism and HPGA reproductive function. The neuronal expression of INS receptors plays an important role in HPGA through its effect on LH secretion. The most important pathological changes in reproductive function caused by HPGA sexual endocrine disorder are hyperandrogenemia and IR. In the model of INS receptor knockout, the secretion of sex hormone is insufficient, leading to infertility and metabolic syndrome. INS receptor has different expression patterns in hypothalamus and pituitary. Nirko mice (INS receptor gene neuron specific knockout mice) cannot maintain the function of Leydig cells due to hypothalamic dysfunction and insufficient GN secretion. Approximately $20 \%$ of seminiferous tubules (STs) lack lumen and have almost no mature spermatogenic cells, indicating that the fertility of Nirko mice is decreased.

The pathogenesis of male infertility and sexual dysfunction caused by abnormal HPGA may be related to the abnormal INS signaling and the dysregulation of kisspeptin expression in hypothalamus (24). G-protein-coupled receptor 54 (GPR54) combined with kisspeptin functions in hypothalamic GnRH neurons to activate the HPGA axis and cause GnRH secretion. Kisspeptin/GPR54 complex is the key to maintain HPGA function and an important factor determining $\mathrm{GnRH}$ secretion $(25,26)$. Kisspeptin is also a "molecular switch" initiating puberty, and its gene mutation leads to hypogonadotropic hypogonadism (HH). The deficiency of INS reduces kisspeptin protein expression in the hypothalamus, resulting in decrease in $\mathrm{GnRH}$ secretion leading to HH. Supplementation of INS can correct the abnormality of GnRH (27). Compared with the peripheral gonads, abnormal levels of INS have a greater impact on hypothalamus and pituitary. Lin et al. (28) demonstrated that the upregulation of kisspeptin expression in the preoptic area can be involved in the stimulation of HPGA function in male rats. A study on the Gnv-3 cell line of GnRH-expressing hypothalamic neurons showed that neurons are INS sensitive tissues and express INS receptors (29). The increased expression of kisspeptin in the preoptic area of the hypothalamus indicates that INS affects the expression of kisspeptin in the Gnv-3 cell line, and the target of rapamycin
(mTOR) pathway has a regulatory effect on kisspeptin mRNA expression and gene transcription. In the central nervous system, GnRH expression is mediated through the mTOR signaling pathway. Activation of mTOR stimulates the expression of kisspeptin and increases the secretion of LH. Rapamycin, an inhibitor of mTOR, can block the above effects. In conclusion, INS regulates kisspeptin protein through the mTOR pathway and affects HPGA function, and $\mathrm{GnRH}$ regulates reproductive function through kisspeptin and kisspeptin neurons.

\section{Effect of Insulin Resistance on Male Reproduction}

Insulin resistance (IR) is an important pathogenic factor and a pathological characteristic of T2DM (10). There is a causal relationship between IR and reproductive hormones: reproductive hormones can affect INS sensitivity and androgens can ameliorate IR and slow down the development of DM. Androgens are closely related to DM (30). T levels are significantly lower in DM patients compared to healthy individuals. The lower the T levels, the higher the incidence rates of DM. IR is the key link between $\mathrm{T}$ and DM. Decreases in androgen promote the development of IR and DM. Dehydroepiandrosterone (DHEA) can reduce serum triglycerides, interleukin-6, TNF- $\alpha$, and other inflammatory factors (31). A prospective cohort study of non-diabetic elderly men shows that the prevalence of DM is lower in patients with higher levels of total $\mathrm{T}$ and free $\mathrm{T}$, and sex hormone binding protein (SHBG) still exists after the effect is removed (32). Several groups have found that the levels of total $\mathrm{T}$, free $\mathrm{T}$, and SHBG in DM patients are significantly lower than those in non-DM patients, and $\mathrm{T}$ and SHBG are negatively correlated with IR $(33,34)$. T promotes glucose uptake, glycolysis, and oxidative phosphorylation. Additionally, $\mathrm{T}$ ameliorates IR by enhancing glucose transport, inhibiting inflammatory response, improving mitochondrial function, and inhibiting adipocyte and tissue proliferation. These indicate that $\mathrm{T}$ plays an important role in maintaining INS sensitivity. In ovariectomized rats, the sensitivity to INS is increased, and androgen supplementation ameliorates IR in and out of the liver (35). There is evidence $(36,37)$ showing that with the increase of homeostatic model assessment of insulin resistance (HOMAIR) index, T presents a downward trend, and the percentage of sperm forward movement shows a downward trend (36, 37). There is no significant difference between $T$ and sperm concentration or total number $(p>0.05)$. HOMA-IR is negatively correlated with serum $\mathrm{T}$ and progesterone (PR) $(p<0.05)$. However, Verit and colleagues (38) observed that HOMA-IR shows no significant correlation with sperm count, motility, and morphology. Zag (zinc alpha glycoprotein) (39) is a new type of adipokines, which plays an important role in alleviating IR. It can enhance INS signal 
transduction and affect the expression of adipokines and inflammatory factors. Zag can be synthesized in epithelial cells and secreted into the semen to affect physiological processes such as fertilization.

\section{Progression in the Treatment of Diabetes Mellitus-Related Male Reproductive Dysfunction}

It is a challenge to treat DM-related male reproductive dysfunction, and blood sugar control is still the key. In the recent years, great efforts have been made to investigate therapeutic approaches for the improvement and treatment of diabetes mellitus erectile dysfunction (DMED). These include stem cell transplantation, gene therapy, vascularization technology, INS treatment, mecobalamin and lipoic therapy, scutellarin (SCU) therapy, acupuncture and moxibustion, androgen therapy, and antioxidant therapy (40-46).

It has been found that stem cells can have a paracrine effect on surrounding tissues, and can also differentiate into a variety of functional cells such as neurons, endothelium, and smooth muscle, thereby repairing damaged tissues caused by DM and improving erectile function (40). Therefore, stem cell transplantation can be a new treatment method for DMED in the clinic.

Gene therapy is also a new direction. The target genes of DMED patients mainly include vascular endothelial growth factor (VEGF) and NOS genes, and the therapy involving these genes can also help improve erectile function. A recent study shows that gene repair therapy activates related cell signal transduction pathways and induces nerve and vascular regeneration (41). In addition, vascularization technology has been used to achieve cavernous smooth muscle angiogenesis and has broad prospects in DMED treatment.

Regarding the treatment of diabetic retrograde ejaculation, blood sugar control is the key, including intensive INS treatment and recovery of pelvic autonomic nerve function. Mecobalamin can repair nerves, and lipoic acid has antioxidant and endothelial functions. Some recent studies have shown that mecobalamin combined with lipoic acid has better effect on the treatment of diabetic neuropathy; however, Ma et al. showed that mecobalamin combined with lipoic acid does not achieve a better therapeutic effect as compared with each agent alone (42). For patients with fertility requirements, in-vitro fertilization, and embryo transfer (IVF-ET) is highly recommended.

Traditional Chinese medicine (TCM)-related therapy is a promising treatment approach for DMED. As the onset of DM is getting younger, reproductive dysfunction caused by DM has gradually attracted attention. Oxidative stress and vascular microcirculation disorders caused by DM can induce cell apoptosis and cause damage to organ function (43), and it is considered to be the main cause of damage to the reproductive system. Therefore, regulating oxidative stress and improving microcirculation are expected to become important means for the prevention and treatment of diabetic complications. Many natural products have significant antioxidant capacity. Breviscapine can be used as a supplement for the prevention or treatment of diabetic complications. Scutellarin (SCU) is another one. Although SCU cannot regulate blood glucose levels, it reduces oxidative stress and has antioxidant effects. SCU improves microcirculation by increasing VEGF expression, reducing blood viscosity, and inhibiting platelet aggregation, thus reducing testicular damage $(44,45)$. Acupuncture has been widely used in the treatment of T2DM in China. It has been shown that acupuncture improves IR and increases glucose metabolism in T2DM rats. It has also been confirmed that acupuncture and moxibustion have antioxidant effects, improving the levels and activity of SOD, protecting the morphology and structure of mitochondria, and improving ATP synthesis in mitochondria.

Androgen therapy is another treatment of choice for DMED. Androgen inhibits inflammatory response, improves mitochondrial function, enhances glucose transport through glut (glucose transporter), improves glucose metabolism, and reduces IR. It has been shown that $\mathrm{T}$ supplementation can slow down the damage of the reproductive system of diabetics.

Lastly, antioxidant therapy has been shown to be effective in the treatment of DMED. Antioxidant therapy reduces OSinduced damage to sperm via stimulating the secretion of FSH and LH as well as T synthesis. Coenzyme Q10 and Nacetylcysteine improve sperm quality by increasing $\mathrm{T}$ and inhibin B; while vitamin $\mathrm{C}$, vitamin $\mathrm{E}$, and glutathione can be used as adjuvant therapy for improving sperm quality. The antioxidant L-carnitine improves the levels of antioxidants in seminal plasma and promotes spermatogenesis and maturation (46). Early antioxidant treatment reduces islet cell damage and promotes islet cell proliferation. Vitamin $\mathrm{C}$ and antioxidant SOD can delay the process of islet cell apoptosis. SOD also improves autonomic nerve conduction velocity and restores endothelial function. It has been demonstrated that increasing zinc intake reduces the severity of type 1 diabetes in animal models, and Zag also reduces IR through a variety of mechanisms.

In general, the treatment of DM-induced male reproduction-related complications requires comprehensive treatment, and blood sugar control is the key strategy. Only when blood sugar is well controlled, it can be possible to prevent peripheral vascular disease and diabetic neuropathy. When diabetic retrograde ejaculation and DMED occur, and testicular tissue structure and semen quality are changed, it is very important to control blood sugar, together with other treatment strategies, such as improving lipid metabolism, protecting nerves and blood vessels, as well as zinc supplementation and related antioxidants, and all these are helpful to improve semen quality and male reproductive 


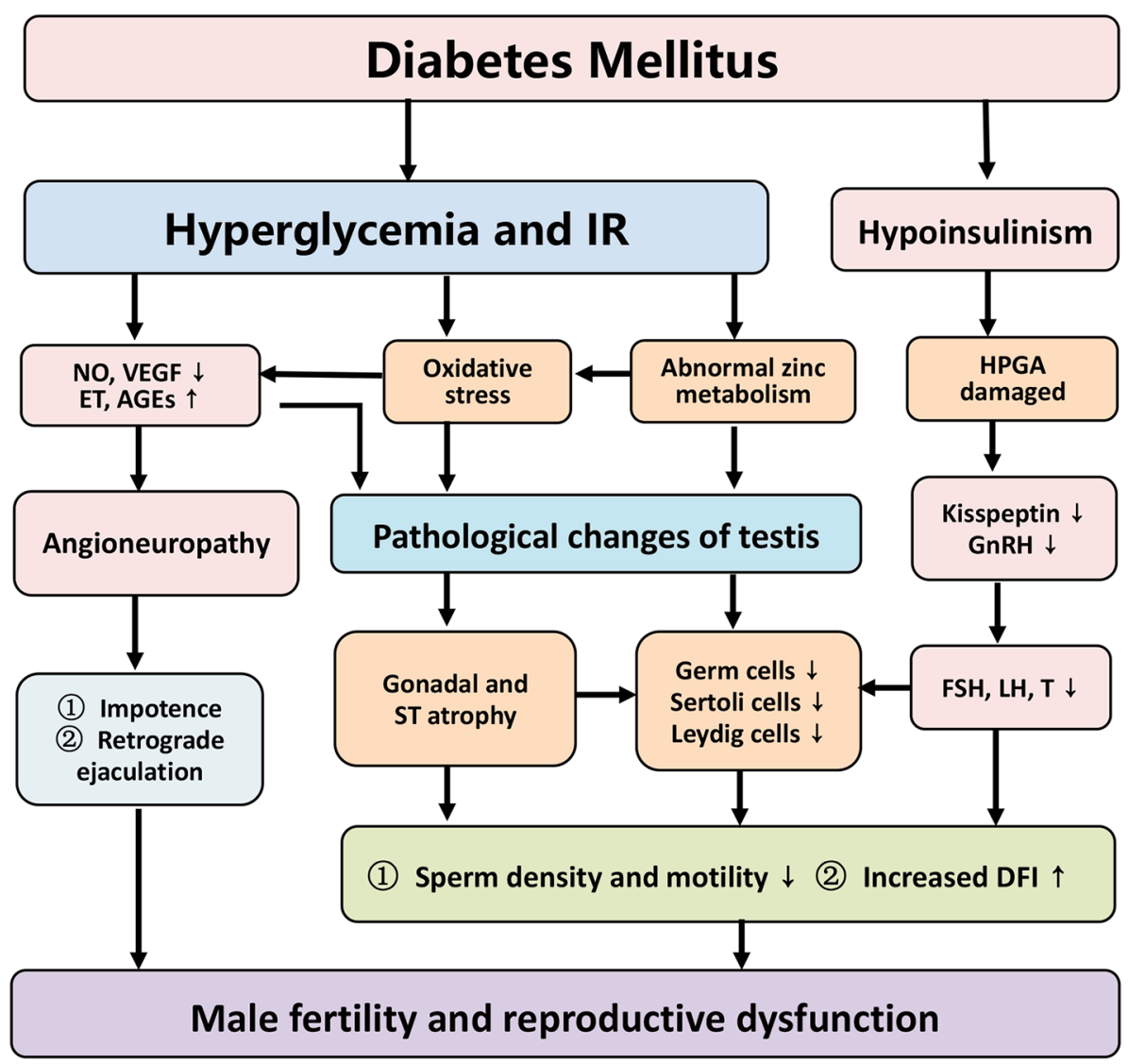

Figure 1. A schematic diagram illustrating a hypothetical model of the impact of diabetes mellitus (DM)-caused metabolic disorder and insulin abnormality on male sexual and reproductive dysfunction. This schematic diagram shows that glucose metabolism disorder and IR in DM cause angioneuropathy through decreases in synthesis and release of NO and VEGF and increases in ET and AGEs in the endothelium leading to ED and retrograde ejaculation. On the other hand, abnormal glucose metabolism can lead to oxidative stress and the loss of zinc. Oxidative stress is a common mechanism underlying diabetic complications, and it also promotes changes in the above processes. Zinc is a strong antioxidant, and zinc deficiency aggravates oxidative stress injury. In addition, insulin deficiency in diabetic patients also causes HPGA damage, thereby reducing secretion of GnRH, FSH, LH, and T and leading to testicular atrophy, stromal cell (Sertoli and Leydig) atrophy, ST damage, and spermatogenic cell damage. All of these factors may act cooperatively to suppress sexual and reproductive function in men. Such a mechanism may serve to integrate the roles of these factors in the reduction of sperm density and motility and increase of deformity rate and sperm DNA fragmentation index, which may underlie the mechanisms through which DM caused male fertility and reproductive dysfunction. See the text for details. IR: Insulin resistance; NO: nitric oxide; VEGF: vascular endothelial growth factor; ET: endothelin; AGE: advanced glycation end product; ED: erectile dysfunction; HPGA: hypothalamic-pituitary-gonadal axis; GnRH: gonadotropin-releasing hormone; FSH: follicle stimulating hormone; LH: luteinizing hormone; T: testosterone; ST: seminiferous tubule; DFI: sperm DNA fragmentation index.

function. Finally, TCM treatment as well as acupuncture and moxibustion can improve microcirculation, protect blood vessels, and eliminate oxygen free radicals. It has been demonstrated that TCM is an effective treatment for DMcaused male reproductive dysfunction.

\section{Conclusion and Future Directions}

DM is a hormonal and metabolic disorder which causes many complications and affects multiple organs and systems in the human body including the male reproductive system. Although the molecular basis of diabetes-caused male sexual and reproductive dysfunction is as yet not completely known, abundant evidence shows that multiple factors and mechanisms are involved in the process (Figure 1). Oxidative stress is a common mechanism underlying diabetic complications. Microcirculation disturbance and oxidative stress are considered to be the main factors causing reproductive system damage. Hyperglycemia leads to the impairment of vascular endothelial function, including the decrease in endothelial NO (eNO) synthesis, the increase of oxidative stress, and the glycosylation of elastic fibers in vascular wall. Finally, hyperglycemia causes peripheral neurovascular diseases, leading to DMED and bladder 
dysfunction. Semen quality is characterized by decreased sperm concentration and motility, as well as high incidence of sperm DNA damage, DNA breakage, and sperm apoptosis. DM causes HPGA damage, reduces hypothalamic GnRH secretion, and decreases plasma LH, FSH, PRL and $\mathrm{T}$, resulting in spermatogenesis disorders. Impaired glucose utilization due to insulin deficiency leads to decreased pituitary protein synthesis and reduced gonadotropin secretion. Zinc has a strong antioxidant effect and plays a key role in scavenging free radicals. Zinc can inhibit lipid oxidation of sperm membrane and maintain the integrity of cell membrane structure. Oxidative stress affects zinc homeostasis and influences the expression of zinc transporters. IR is an important pathogenic factor and a pathological characteristic of T2DM. IR leads to erectile dysfunction and hypogonadism. Androgen ameliorates IR by increasing glucose transport, improving mitochondrial function, inhibiting inflammatory response, and suppressing the proliferation of adipocytes and adipoprogenitor cells, all of which slow down the development of DM.

Accumulating evidence shows that oxidative stress plays a very important role in the occurrence and development of $\mathrm{DM}$, and therefore it is of great significance for the prevention and treatment of diabetes-related male reproductive dysfunction. There are still many key scientific issues regarding the mechanism regulating oxidative stress, including how cells perceive oxidative stress, the ROS threshold for activating key antioxidant molecules, and the effects of other forms of stress (such as metabolic stress, hypoxia, and inflammation) on oxidative stress response. The molecular mechanism of inhibition of INS secretion by oxidative stress provides multiple candidate targets for the treatment of DM. Key factors (such as p38 mitogenactivated protein kinases and c-Jun N-terminal kinase 1) in the process of INS tolerance induced by oxidative stress can reverse oxidative stress-induced INS tolerance, suggesting that these factors can be potential targets for the treatment of DM. The unfolded protein response (UPR) is a cellular stress response related to the endoplasmic reticulum stress. It has been found that UPR regulates the pathogenesis of IR and DM by affecting inflammation and lipid metabolism in the hypothalamus and is closely related to the maintenance of islet $\beta$-cell function. It provides a theoretical basis for the prevention and treatment of DM by targeting IRE1, a key factor in the pathogenesis of DM. Therefore, future research should screen and identify more potential drug targets based on further understanding of DM. In summary, as more and more mechanisms of action of oxidative stress in DM are unveiled, our understanding of the impact of DM on male reproduction will increase, and new and better approaches to improve reproductive health in men and to treat DM-caused male sexual and reproductive dysfunction will be developed.

\section{Conflicts of Interest}

The Authors declare that they do not have any competing interests regarding this article.

\section{Authors' Contributions}

$\mathrm{ZH}$ and GY were involved in the design of the work, literature search, analysis and interpretation of data, and drafted the article. QQL, QZ, and JD supervised the study and were involved in the critical revision of the article. JD conceived the project and obtained the funding. All Authors have approved the final version of the article.

\section{Acknowledgements}

This work was supported by a grant for Omics evaluation and high precision genetic screening of embryo implantation potential in officers and soldiers of the PLA (No. 17js008) and a grant by Guilin Scientific Research and Technology Development Plan (No. 20180106-4-7). The Authors would also like to thank Diane Cooper, NIH Library, for reviewing the article.

\section{References}

1 Castela Â and Costa C: Molecular mechanisms associated with diabetic endothelial-erectile dysfunction. Nat Rev Urol 13(5): 266-274, 2016. PMID: 26878803. DOI: 10.1038/nrurol.2016.23

2 Li Y, Liu L, Li F, Zhang X, Zhang B and Wang Y: Study and treatment of male erectile dysfunction induced by diabetes mellitus. Chin J Hum Sex 27(03): 10-13, 2018 (in Chinese). DOI: $10.3969 /$ j.issn.1672-1993.2018.03.002. Available at: https://d.wanfangdata.com.cn/periodical/ChlQZXJpb2RpY2FsQ0 hJTmV3UzIwMjEwNjA4Eg56Z3hreDIwMTgwMzAwMhoIeHA 5NGpkd2o\%3D [Last accessed on June 14, 2021]

3 Wang XM, Song SS, Xiao H, Gao P, Li XJ and Si LY: Fibroblast growth factor 21 protects against high glucose induced cellular damage and dysfunction of endothelial nitric-oxide synthase in endothelial cells. Cell Physiol Biochem 34(3): 658-671, 2014. PMID: 25170746. DOI: 10.1159/000363031

4 Zhao W, Hu Y, Long L, Cai B and Li Y: Protective effects of scutellarin on testicular vessels of rat with type II diabetes mellitus-induced testicular damages. J Sun Yat-Sen Univ Med Sci 41(02): 260-267, 2020 (in Chinese). DOI: 10.13471/ j.cnki.j.sun.yat-sen.univ(med.sci).2020.0035. Available at: https://d.wanfangdata.com.cn/periodical/ChlQZXJpb2RpY2FsQ0 hJTmV3UzIwMjEwNjA4EhF6c31rZHh4YjIwMjAwMjAxMxoId GYyZWd3eHo\%3D [Last accessed on June 14, 2021]

5 Liu W, Ma J, Han R and Wang S: Expression of zinc transporter in gonads of diabetic male rats. Chin J Androl 34(5), 2020 (in Chinese). DOI: 10.3969/j.issn.1008-0848.2020.05.002. Available at: https://d.wanfangdata.com.cn/periodical/ChlQZXJpb2RpY2 FsQ0hJTmV3UzIwMjEwNjA4EhB6Z25reHp6MjAyMDA1MDA yGggyazh6dDk1bw\%3D\%3D [Last accessed on June 14, 2021]

6 Imani M, Talebi AR, Fesahat F, Rahiminia T, Seifati SM and Dehghanpour F: Sperm parameters, DNA integrity, and protamine expression in patients with type II diabetes mellitus. J Obstet Gynaecol 41(3): 439-446, 2021. PMID: 32705912. DOI: $10.1080 / 01443615.2020 .1744114$ 
7 Maresch CC, Stute DC, Alves MG, Oliveira PF, de Kretser DM and Linn T: Diabetes-induced hyperglycemia impairs male reproductive function: a systematic review. Hum Reprod Update 24(1): 86-105, 2018. PMID: 29136166. DOI: 10.1093/ humupd/dmx033

8 Shi GJ, Li ZM, Zheng J, Chen J, Han XX, Wu J, Li GY, Chang Q, Li YX and Yu JQ: Diabetes associated with male reproductive system damages: Onset of presentation, pathophysiological mechanisms and drug intervention. Biomed Pharmacother 90: 562-574, 2017. PMID: 28407577. DOI: 10.1016/j.biopha. 2017.03.074

9 Deng P, Ma J, Han R, Chen Z, Zhou J, Zhang Y and Wang S: Clinical study of the influence of reproductive endocrine hormone, serum levels of ET and NO on these men quality of diabetic males. Chin J Fam Plan 27(01): 21-24, 2019 (in Chinese). DOI: 10.3969/j.issn.1004-8189.2019.01.005. Available at: https://d.wanfangdata.com.cn/periodical/ChlQZXJpb2RpY2 FsQ0hJTmV3UzIwMjEwNjA4EhJ6Z2poc314enoyMDE5MDEw MDYaCHA3YzZyYmVj [Last accessed on June 14, 2021]

10 Kaur G, Thompson LA, Babcock RL, Mueller K and Dufour JM: Sertoli cells engineered to express insulin to lower blood glucose in diabetic mice. DNA Cell Biol 37(8): 680-690, 2018. PMID: 29927618. DOI: 10.1089/dna.2017.3937

11 Cao H, Ha X, Li X, Xu Q, Bai Y and Zeng T: Molecular mechanism of free fatty acids-induced insulin resistance. Med J Chin PLA 42(01): 81-85, 2017 (in Chinese). DOI: 10.11855/j.issn.0577-7402.2017.01.16. Available at: https://d wanfangdata.com.cn/periodical/ChlQZXJpb2RpY2FsQ0hJTmV3 UzIwMjEwNjA4EhBqZmp5eHp6MjAxNzAxMDE2GghhcGF1 NmdjZw\%3D\%3D [Last accessed on June 14, 2021]

12 Yang X, Xing H, Liu J, Yang L, Ma H and Ma H: MicroRNA 802 increases hepatic oxidative stress and induces insulin resistance in high fat fed mice. Mol Med Rep 20(2): 1230-1240, 2019. PMID: 31173239. DOI: 10.3892/mmr.2019.10347

13 Sahin N, Orhan C, Erten F, Tuzcu M, Defo Deeh PB, Ozercan IH, Juturu V and Kazim S: Effects of allyl isothiocyanate on insulin resistance, oxidative stress status, and transcription factors in high-fat diet/streptozotocin-induced type 2 diabetes mellitus in rats. J Biochem Mol Toxicol 33(7): e22328, 2019 PMID: 30927557. DOI: 10.1002/jbt.22328

14 Dong P, Zhang C and Tao M: Relationship between peripheral neuropathy and lower extremity vascular disease in type 2 diabetes mellitus. Chin J Clin Res 30(06): 744-747, 2017 (in Chinese). DOI: 10.13429/j.cnki.cjcr.2017.06.007. Available at: https://d.wanfangdata.com.cn/periodical/ChlQZXJpb2RpY2FsQ0 hJTmV3UzIwMjEwNjA4Eg96Z2NreXgyMDE3MDYwMDcaC G92ZTViZ2Yy [Last accessed on June 14, 2021]

15 Song $H$, Wang $M$ and Xin T: Mst1 contributes to nasal epithelium inflammation via augmenting oxidative stress and mitochondrial dysfunction in a manner dependent on $\mathrm{Nrf} 2$ inhibition. Journal of Cellular Physiology 234(12): 23774-23784, 2021. DOI: $10.1002 / j c p .28945$

16 Usui T, Kajita K, Kajita T, Mori I, Hanamoto T, Ikeda T, Okada H, Taguchi K, Kitada Y, Morita H, Sasaki T, Kitamura T, Sato T, Kojima I and Ishizuka T: Elevated mitochondrial biogenesis in skeletal muscle is associated with testosterone-induced body weight loss in male mice. FEBS Lett 588(10): 1935-1941, 2014. PMID: 24726723. DOI: 10.1016/j.febslet.2014.03.051

17 Wang F, Yang J, Sun J, Dong Y, Zhao H, Shi H and Fu L: Testosterone replacement attenuates mitochondrial damage in a rat model of myocardial infarction. J Endocrinol 225(2): 101111, 2015. PMID: 25770118. DOI: 10.1530/JOE-14-0638

18 Taravati A and Tohidi F: Association between seminal plasma zinc level and asthenozoospermia: a meta-analysis study. Andrologia 48(6): 646-653, 2016. PMID: 26541500. DOI: 10.1111/and.12494

19 Jurowski K, Szewczyk B, Nowak G and Piekoszewski W: Biological consequences of zinc deficiency in the pathomechanisms of selected diseases. J Biol Inorg Chem 19(7): 1069-1079, 2014. PMID: 24748223. DOI: 10.1007/s00775-0141139-0

20 Fung EB, Gildengorin G, Talwar S, Hagar L and Lal A: Zinc status affects glucose homeostasis and insulin secretion in patients with thalassemia. Nutrients 7(6): 4296-4307, 2015. PMID: 26043030. DOI: 10.3390/nu7064296

21 Cai Y, Kirschke CP and Huang L: SLC30A family expression in the pancreatic islets of humans and mice: cellular localization in the $\beta$-cells. J Mol Histol 49(2): 133-145, 2018. PMID: 29372370. DOI: $10.1007 / \mathrm{s} 10735-017-9753-0$

22 Zhong M, Jia B, Liu H, Wang Z, Wang Y and Chi Z: ZnT8 is involved in testosterone synthesis in mouse Leydig cells. Prog Anat Sci 18(01): 12-14, 2012 (in Chinese). DOI: 10.16695/ j.cnki.1006-2947.2012.01.012. Available at: https://kns.cnki.net/ $\mathrm{kcms} /$ detail/detail. asp $x$ ?dbcode $=$ CJFD $\&$ dbname $=$ CJFD2012 \&fil ename $=J P K X 201201004 \& v=\% 25 \mathrm{mmd} 2 \mathrm{BuZdY} 08 \% 25 \mathrm{mmd} 2 \mathrm{~F} 78$ 1fDIOfkJ\%25mmd2FtwcYz6IuELY\%25mmd2FhK6DpLuFj1jgeI 9UAyK1QLP7AtLLFueC8 [Last accessed on June 14, 2021]

23 Sahu SK, Cockrem JF, Parkinson TJ and Laven RA: Effects of $\mathrm{GnRH}$, a progesterone-releasing device, and energy balance on an oestrus synchronisation program in anoestrous dairy cows. Aust Vet J 95(8): 281-288, 2017. PMID: 28749022. DOI: 10.1111/avj.12611

24 Liu J and Tang J: Effects of pomegranate extract in supplementing gonadotropin-releasing hormone therapy on idiopathic central precocious puberty in Chinese girls: a randomized, placebo-controlled, double-blind clinical trial. Food Funct 8(2): 695-700, 2017. PMID: 28091641. DOI: 10.1039/ c6fo01616b

25 Brown RJ, Joseph J, Cochran E, Gewert C, Semple R and Gorden P: Type B insulin resistance masquerading as ovarian hyperthecosis. J Clin Endocrinol Metab 102(6): 1789-1791, 2017. PMID: 27911591. DOI: 10.1210/jc.2016-3674

26 Baldauff NH and Witchel SF: Polycystic ovary syndrome in adolescent girls. Curr Opin Endocrinol Diabetes Obes 24(1): 5666, 2017. PMID: 27906711. DOI: 10.1097/MED.000000000 0000309

27 Öztin H, Çağıltay E, Çağlayan S, Kaplan M, Akpak YK, Karaca $\mathrm{N}$ and Trğlıŏlu $\mathrm{M}$ : Kisspeptin levels in idiopathic hypogonadotropic hypogonadism diagnosed male patients and its relation with glucose-insulin dynamic. Gynecol Endocrinol 32(12): 991-994, 2016. PMID: 27616469. DOI: 10.1080/ 09513590.2016 .1214258

28 Lin J: Molecular mechanism of spermatogenic disorder in immune orchitis and insulin regulate hypothalamus-pituitarygonadal axis function. Peking Union Med Coll, 2018 (in Chinese). DOI: 10.7666/d.Y3514107. Available at: https://d.wanfangdata.com.cn/thesis/ChJUaGVzaXNOZXdTMjA yMTA1MTkSCFkzNTE0MTA3Ggg0ZWU5bTVkdw\%3D\%3D [Last accessed on June 14, 2021]

29 Hannan MA, Kawate N, Fukami Y, Weerakoon WW, Büllesbach $\mathrm{EE}$, Inaba $\mathrm{T}$ and Tamada $\mathrm{H}$ : Effects of long-acting $\mathrm{GnRH}$ 
antagonist, degarelix acetate, on plasma insulin-like peptide 3 , testosterone and luteinizing hormone concentrations, and scrotal circumference in male goats. Theriogenology 88: 228-235, 2017. PMID: 27793455. DOI: 10.1016/j.theriogenology.2016.09.032

30 Lorigo M, Mariana M, Oliveira N, Lemos MC and Cairrao E: vascular pathways of testosterone: clinical implications. J Cardiovasc Transl Res 13(1): 55-72, 2020. PMID: 31820333 DOI: 10.1007/s12265-019-09939-5

31 Cai X, Tian Y, Wu T, Cao CX, Li H and Wang KJ: Metabolic effects of testosterone replacement therapy on hypogonadal men with type 2 diabetes mellitus: a systematic review and metaanalysis of randomized controlled trials. Asian J Androl 16(1): 146-152, 2014. PMID: 24369149. DOI: 10.4103/1008$682 X .122346$

32 Salminen M, Vahlberg T, Räihä I, Niskanen L, Kivelä SL and Irjala $\mathrm{K}$ : Sex hormones and the risk of type 2 diabetes mellitus: A 9-year follow up among elderly men in Finland. Geriatr Gerontol Int 15(5): 559-564, 2015. PMID: 24891075. DOI: 10.1111/ggi.12312

33 Zhang J, Huang X, Liao M, Gao Y, Tan A, Yang X, Zhang H, Mo L, Zhang Y, Lu Z, Wu C, Hu Y and Mo Z: Both total testosterone and sex hormone-binding globulin are independent risk factors for metabolic syndrome: results from Fangchenggang Area Male Health and Examination Survey in China. Diabetes Metab Res Rev 29(5): 391-397, 2013. PMID: 23463406. DOI: 10.1002/dmrr.2405

34 Cree-Green M, Newcomer BR, Coe G, Newnes L, Baumgartner A, Brown MS, Pyle L, Reusch JE and Nadeau KJ: Peripheral insulin resistance in obese girls with hyperandrogenism is related to oxidative phosphorylation and elevated serum free fatty acids. Am J Physiol Endocrinol Metab 308(9): E726-E733, 2015. PMID: 25714677. DOI: 10.1152/ajpendo.00619.2014

35 Xia F, Xu X, Zhai H, Meng Y, Zhang H, Du S, Xu H, Wu H and Lu Y: Castration-induced testosterone deficiency increases fasting glucose associated with hepatic and extra-hepatic insulin resistance in adult male rats. Reprod Biol Endocrinol 11: 106, 2013. PMID: 24238614. DOI: 10.1186/1477-7827-11-106

36 Ma J, Han RY, Mei XA, Qi YN, Ma JY, Liu WJ and Wang SS: [Correlation of insulin resistance with male reproductive hormone levels and semen parameters]. Zhonghua Nan Ke Xue 24(8): 695-699, 2018. PMID: 30173427.

$37 \mathrm{Hu}$ M and Ma Z: Influence analysis of insulin resistance on male sperm quality. J Modern Med Health 33(08): 1158-1160, 2017. DOI: 10.3969/j.issn.1009-5519.2017.08.013. Available at: https://d.wanfangdata.com.cn/periodical/ChlQZXJpb2RpY2FsQ0 hJTmV3UzIwMjEwNjA4Eg94ZH15d3MyMDE3MDgwMTMaC HdyZmx5aWJ4 [Last accessed on June 14, 2021]

38 Verit A, Verit FF, Oncel H and Ciftci H: Is there any effect of insulin resistance on male reproductive system? Arch Ital Urol Androl 86(1): 5-8, 2014. PMID: 24704923. DOI: 10.4081/ aiua.2014.1.5
39 Guo Y and Zhang S: Research progress in relationship between zine- $\alpha$ 2-glycoprotein and insulin resistance GUO. Chin J Diabetes 28(10): 788-790, 2020. DOI: 10.3969/j.issn.10066187.2020.10.014. Available at: https://d.wanfangdata.com.cn/ periodical/ChlQZXJpb2RpY2FsQ0hJTmV3UzIwMjEwNjA4Eh B6Z3RuYnp6MjAyMDEwMDE0Ggg4cjN5MmM5Yw\%3D\%3 D [Last accessed on June 14, 2021]

40 Khera M, Albersen M and Mulhall JP: Mesenchymal stem cell therapy for the treatment of erectile dysfunction. J Sex Med 12(5): 1105-1106, 2015. PMID: 25974235. DOI: 10.1111/ jsm. 12871

41 Chen X, Huang H, Zhu X, Chen G, Li P, Song F, Xiang C, Guo Y, Zhang S and Zhang X: Focused low-intensity extracorporeal shock wave therapy for erectile dysfunction: Preliminary observation of 32 cases. Nat J Androl 24(06): 529-532, 2018. DOI: 10.13263/j.cnki.nja.2018.06.009. Available at: https:// d.wanfangdata.com.cn/periodical/ChlQZXJpb2RpY2FsQ0hJTm V3UzIwMjEwNjA4Eg56aG5reDIwMTgwNjAxMBoIaGoyMjE3 NTk\%3D [Last accessed on June 14, 2021]

$42 \mathrm{Ma} \mathrm{Z}$ : Treatment strategy of infertility due to retrograde ejaculation induced by diabetes mellitus. Med Forum 20(36): 5075-5077, 2016. DOI: 10.3969/j.issn.1672-1721.2016.36.005. Available

at: https://d.wanfangdata.com.cn/periodical/ChlQZXJpb2RpY2FsQ0 hJTmV3UzIwMjEwNjA4Eg9qY314bHQyMDE2MzYwMDUaC GtsNDJ6N2xl [Last accessed on June 14, 2021]

43 Qadir MMF, Klein D, Álvarez-Cubela S, Domínguez-Bendala J and Pastori RL: The role of microRNAs in diabetes-related oxidative stress. Int J Mol Sci 20(21): 5423, 2019. PMID: 31683538. DOI: $10.3390 /$ ijms20215423

$44 \mathrm{Kim} \mathrm{M}$ and Lee BC: Therapeutic effect of Scutellaria baicalensis on L-thyroxine-induced hyperthyroidism rats. Evid Based Complement Alternat Med 2019: 3239649, 2019. PMID: 31636683. DOI: $10.1155 / 2019 / 3239649$

45 Cui X, Qian DW, Jiang S, Shang EX, Zhu ZH and Duan JA: Scutellariae Radix and Coptidis Rhizoma improve glucose and lipid metabolism in T2DM rats via regulation of the metabolic profiling and MAPK/PI3K/Akt signaling pathway. Int J Mol Sci 19(11): 3634, 2018. PMID: 30453687. DOI: 10.3390/ ijms 19113634

$46 \mathrm{Li}$ Y, Jin L and Zhou Y: Research progress of oxidative stress on male infertility. Chin J Gen Pract 18(10): 1729-1734, 2020. DOI: 10.16766/j.cnki.issn.1674-4152.001605. Available at: https://d.wanfangdata.com.cn/periodical/ChlQZXJpb2RpY2FsQ0 hJTmV3UzIwMjEwNjA4Eg9zeXFreXgyMDIwMTAwMzIaCHd vNWlnY2Y5 [Last accessed on June 14, 2021]

Received May 1, 2021

Revised June 9, 2021

Accepted June 15, 2021 